\title{
Focal plane tuning in wide-field-of-view microscope with Talbot pattern illumination
}

\author{
Jigang $\mathbf{W u},{ }^{1,2, *}$ Guoan Zheng, ${ }^{1}$ Zheng Li, ${ }^{1}$ and Changhuei Yang, ${ }^{1,3}$ \\ ${ }^{1}$ Department of Electrical Engineering, California Institute of Technology, 1200 East California Boulevard, \\ Pasadena, California 91125, USA \\ ${ }^{2}$ University of Michigan-Shanghai Jiao Tong University Joint Institute, Shanghai Jiao Tong University, \\ Shanghai 200240, China \\ ${ }^{3}$ Department of Bioengineering, California Institute of Technology, 1200 East California Boulevard, \\ Pasadena, California 91125, USA \\ *Corresponding author: jigang@Caltech.edu
}

Received April 15, 2011; accepted May 1, 2011; posted May 10, 2011 (Doc. ID 145985); published June 6, 2011

\begin{abstract}
We have developed a focal plane tuning technique for use in focus-grid-based wide-field-of-view microscopy (WFM). In WFM, the incidence of a collimated beam on a mask with a two-dimensional grid of aperture produced the Talbot images of the aperture grid. The Talbot pattern functioned as a focus grid and was used to illuminate the sample. By scanning the sample across the focus grid and collecting the transmission, we can generate a microscopy image of the sample. By tuning the wavelength of the laser, we can tune the focal plane of the WFM and acquire images of different depth into the sample. Images of a green algae microscope slide were acquired at different focal planes for demonstration. (c) 2011 Optical Society of America

OCIS codes: $170.0110,050.1970,170.5810$.
\end{abstract}

The ability to collect wide-field-of-view (FOV) microscopic images is highly desired in many applications, such as digital pathology [1] or high-throughput screening [2]. There are numerous recent efforts aimed at addressing this need [3,4]. Previously, we have developed a wide-FOV microscope based on holographic focus grid illumination [5]. In that system, the sample is illuminated with a holographically generated grid of focused light spots, and the transmission of the light spots through the sample is projected onto an imaging sensor by a simple collection optics arrangement. A wide-FOV image can then be acquired by scanning the sample across the focus grid. Unlike conventional microscopy or other multibeam microscopy [6], the resolution and FOV of such a system are not cross-competing design constraints. Here, the resolution is fundamentally set by the light spot size, and the FOV is set by the size of the holographic grid. As long as the collected light spot transmissions are well separated on the imaging sensor (determined by the spot separation and the collection optics), such a system can perform high-resolution wide-FOV microscopy imaging effectively.

In this Letter we demonstrate an alternate microscope design where the focus grid is generated via the Talbot effect. Here, a mask with an aperture grid is illuminated with a collimated laser beam to produce the focus grid. A "self-image" of the aperture grid will be reproduced at integer increments of the Talbot distance [7]. This focus grid replacement has several advantages. First, the focus grid generation is more repeatable and robust because the mask can be readily fabricated by the microfabrication technique. Second, unlike the holographic method, there is no zero-order transmission of the incident beam in the Talbot effect. This will enhance the contrast of the foci on the sensor and thus the signal-to-noise ratio of the image. Third, the focused light spots are more uniform in their powers (spot-to-spot comparison). Finally, this approach allows us to accomplish $z$-axis scanning (focal plane change) by simply tuning the light source wavelength.

This Letter is structured as follows. We will briefly summarize the principle of the Talbot effect and point out certain subtleties that we have to closely consider in adapting the phenomenon for our specific applications. Next, we will describe our experimental setup. We will then report on our demonstration of focal plane tuning and apply the system to perform imaging.

The Talbot effect [8] is a well-known phenomenon in which the transmission from a mask with a periodic grid of apertures will reproduce the grid pattern at regular distance intervals from the mask. The distance interval, termed the Talbot distance $Z_{T}$, can be calculated as

$$
Z_{T}=2 d^{2} / \lambda,
$$

where $d$ is the period of the pattern and $\lambda$ is the wavelength. The periodic pattern will reproduce itself at the distance $Z=m Z_{T}$ or a phase-reversed image at $Z=$ $(m-1 / 2) Z_{T}$, where $m$ is an integer. In principle, any of these planes can be used as the focus grid for our wide-FOV microscope. In our experiment, we used the second phase-reversed Talbot plane $\left(Z_{1.5}=1.5 Z_{T}\right)$ for more convenient experimental setup.

Interestingly, Eq. (1) implies that $Z_{T}$ is inversely proportional to the wavelength. For small tuning of the wavelength, i.e., $\Delta \lambda \ll \lambda$, the Talbot distance change can be calculated as

$$
\Delta Z_{T}=-2 d^{2} \Delta \lambda / \lambda^{2} .
$$

For a periodicity $d=30 \mu \mathrm{m}$ and a nominal wavelength of $700 \mathrm{~nm}$, this implies that we can accomplish a significant plane shift of $55 \mu \mathrm{m}$ at $Z=Z_{1.5}$ for a small wavelength change of $10 \mathrm{~nm}$. This tuning ability can be used in our microscope design to accomplish the nonmechanical focal plane shift. 
As the resolution of our microscopy system depends on the grid spot size generated, the spot size that can be created by the Talbot effect is of importance to us. It is worth noting that the "self-imaging" ability of the Talbot effect is only a paraxial approximation [9]. In other words, the Talbot effect will "self-image" the holes in the mask well only if the hole diameter is much larger than $\lambda[10,11]$. The effect will fail and result in "selfimage" spots that are substantially larger if the hole diameter is comparable to $\lambda$.

As we desire tightly focused light spots for our microscopy application, a straight application of the Talbot effect would not work well. Fortunately, we found that the Talbot field propagation will create tight light spots in planes that are slightly above the Talbot planes. Figures 1(a) and 1(b) show numerical simulations versus experimental characterization of the evolving Talbot patterns around $Z=Z_{1.5, \lambda 1}$ for $\lambda_{1}=702 \mathrm{~nm}$. Our Talbot mask has a periodicity of $30 \mu \mathrm{m}$ and aperture sizes of $800 \mathrm{~nm}$. Figure 1(a) is the simulation results, and Fig. 1(b) shows experimental spots observed under a conventional microscope with a $60 \times$ objective. The simulation was performed by the angular spectrum propagation using Fourier transforms. The $Z$ positions and $w$, the FWHM, of spots except those in the last column are indicated in the figure. The last column corresponds to $Z=Z_{1.5}$. As we can see, the Talbot effect fails to generate a good focal spot at this position (the self-imaging approximation is not valid for this regime).

We further experimentally imaged the spot pattern at wavelength of $\lambda_{2}=692 \mathrm{~nm}$ [Fig. 1(c)]. It is worth noting that the patterns closely resemble the original set. This is not surprising as the wavelength shift is small. It is further worth noting that the entire pattern set has shifted by a distance of $55 \mu \mathrm{m}\left(Z=Z_{1.5, \lambda 2}-Z_{1.5, \lambda 1}=\right.$ $55 \mu \mathrm{m})$ away from the mask. This shift is consistent with the prediction from Eq. (2).

Figure 1 also shows that the light pattern generally consists of a bright central spot and associated concentric rings. As the $Z$ position increases, the center spot gets brighter and larger, while the concentric rings get smaller and finally disappear. By observing the evolving spots, we can see that the best focus spots for imaging are those around $Z_{1.5}-90 \mu \mathrm{m}$. For smaller $z$ positions, the center spot is too weak for a good contrast imaging. While for larger $Z$ positions, the center spot is too large to provide high-resolution images. The observed spots

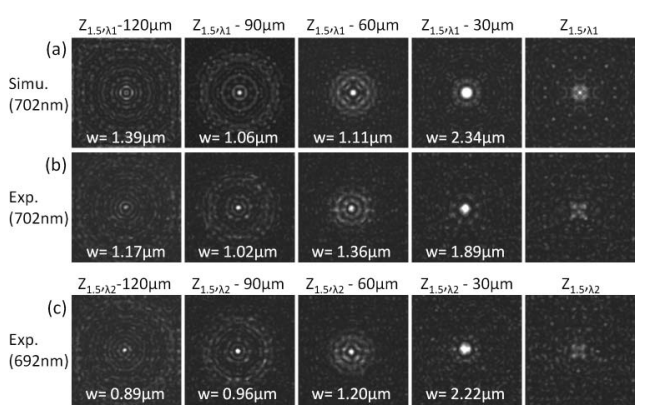

Fig. 1. Evolving of Talbot patterns around $Z=Z_{1.5}$. (a) Simulation spots for $\lambda_{1}=702 \mathrm{~nm}$. (b) Spots for $\lambda_{1}=702 \mathrm{~nm}$. (c) Spots for $\lambda_{2}=692 \mathrm{~nm}$. $w$ is the FWHM spot size, and $Z_{1.5, \lambda 2}-Z_{1.5, \lambda 1}=$ $55 \mu \mathrm{m}$. The size of each pattern figure is $30 \mu \mathrm{m} \times 30 \mu \mathrm{m}$.

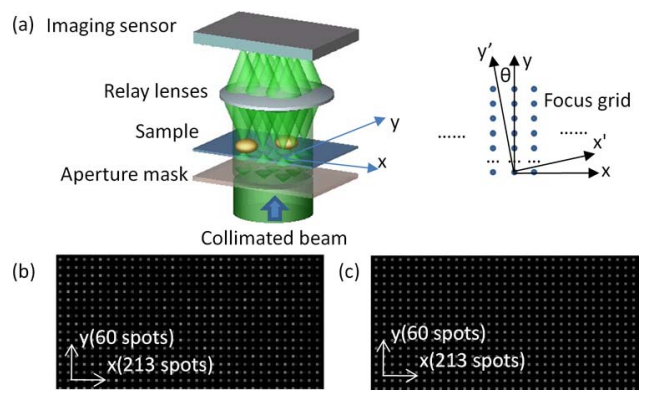

Fig. 2. (Color online) (a) Experimental setup of the wide-FOV microscope based on the Talbot illumination. (b) Part of the aperture grid on the mask plane. (c) Part of the focus grid at $Z=Z_{15}$. The number of spots along the $x$ and $y$ directions for the full mask is indicated.

show that we can get a resolution of $\sim 1 \mu \mathrm{m}$, which was verified by acquiring images of a United States Air Force (USAF) target in the experiment.

Our experimental setup is shown in Fig. 2(a). An aperture mask was illuminated by a collimated beam introduced from a tunable Ti:sapphire laser (SpectraPhysics). As mentioned earlier, we employed an aperture mask with periodicity of $d=30 \mu \mathrm{m}$ and aperture size of $800 \mathrm{~nm}$. The laser wavelength was tuned from 692 to $702 \mathrm{~nm}$ to vary the Talbot distance. The distance between the mask and the sample was set at $Z=Z_{1.5}$ during initial alignment. We subsequently fine-tuned the distance to make use of the tighter light spots discussed in the previous paragraph.

According to Eq. (2), the tuning range of the focal plane position was $55 \mu \mathrm{m}$ in the experiment. Similar to the setup in our previous paper [3], the sample was illuminated by the focus grid and the transmission of the foci was projected by relay lenses (NT45-760, Edmund Optics, Inc.) onto an imaging sensor (LuCam080M, Lumenera Corp.). The sample was scanned across the focus grid and the scanning direction ( $y^{\prime}$ direction) was slightly tilted at a small angle $\theta$ with respect to the focus grid, which was oriented at the $x$ and $y$ directions. The image can be reconstructed by combining line scans from each focal spots and properly shifting each line according to the scanning speed.

Figures 2(b) and 2(c) show part of the aperture grid on the mask plane and part of the focus grid at $Z=Z_{1.5}$, respectively. We can see that the intensity uniformity of the focus grid is better than the original aperture grid. The averaged relative adjacent spot intensity variation is $4 \%$ for the focus grid and $20 \%$ for the original aperture grid. This is reasonable, because many apertures will contribute to a focal spot; thus, the intensity difference of the original aperture grid will be partially averaged out. During image processing, we further ensure image uniformity by normalizing each scan line to compensate for the nonuniformity intensity distribution of the focal spots in the experiment.

We next used our system for imaging demonstration. The focus grid we used has $213 \times 60$ (in the $x$ and $y$ directions, respectively) spots, and the scanning was performed along the $y^{\prime}$ direction. Thus the reconstructed image is oriented at the $x^{\prime}$ and $y^{\prime}$ directions. The image will have a sawtooth shape at the starting and ending part because of the linear scanning characteristics. 


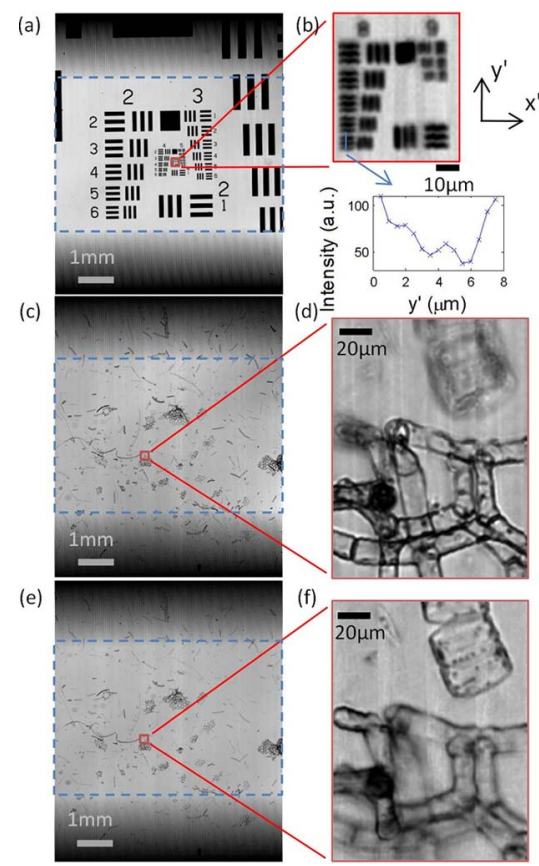

Fig. 3. (Color online) Images acquired by the Talbot wide-FOV microscope with an effective FOV of $6.4 \mathrm{~mm} \times 4.2 \mathrm{~mm}$. (a) Wide-FOV image of a USAF target, with effective FOV indicated in the large dashed rectangle. (b) Expanded view of the smallest feature of the target and cross-sectional profile of group 8, element 6. (c) Wide-FOV image of a mixed green algae slide with $\lambda=692 \mathrm{~nm}$, with effective FOV indicated in the large dashed rectangle. (d) Expanded view of the region indicated in (c). (e) Wide-FOV image of a mixed green algae slide with $\lambda=702 \mathrm{~nm}$, with effective FOV indicated in the large dashed rectangle. (f) Expanded view of the region indicated in (e).

The effective FOV in $x^{\prime}$ direction is $213^{*} d \approx 6.4 \mathrm{~mm}$. In the experiments, the sample was moved at a speed of $v=0.165 \mathrm{~mm} / \mathrm{s}$ for $L=6 \mathrm{~mm}$. At the same time, the imaging sensor acquired 12,000 frames at $F=330$ frames/s. The exposure time of the sensor was $0.5 \mathrm{~ms}$. Thus, the sampling distance in the $y^{\prime}$ direction is $v / F=0.5 \mu \mathrm{m}$. The sampling distance in the $x^{\prime}$ direction is determined by the angle $\theta$ between the scanning direction ( $y^{\prime}$ direction) and the grid orientation ( $y$ direction), which was set to be 0.0167 . Thus the sampling distance in $x^{\prime}$ direction is $d^{*} \sin (\theta)=0.5 \mu \mathrm{m}$. The effective FOV in the $y^{\prime}$ direction can be calculated by $L-H=4.2 \mathrm{~mm}$, where $H=60^{*} d=$ $1.8 \mathrm{~mm}$ is the extent of the focus grid in the $y$ direction.

For the resolution test, we first image a USAF target, as shown in Figs. 3(a) and 3(b). Figure 3(a) is a wide-FOV image $(6.4 \mathrm{~mm} \times 4.2 \mathrm{~mm})$, and Fig. $3(\bar{b})$ is the expanded view of the region indicated in Fig. 3(a). The crosssectional profile of group 8, element 6 is also shown in the figure. We can see that features sized with a line width of $1.1 \mu \mathrm{m}$ (group 8, element 6) can be resolved, which agrees with the measured spot size of $\sim 1 \mu \mathrm{m}$ as shown in Fig. 1.

We then acquired images of a mixed green algae microscope slide (Carolina Biological Supply Company). Images were acquired at different focal planes, where Fig. 3(c) is for $\lambda=692 \mathrm{~nm}$ and Fig. 3(e) is for $\lambda=$ $702 \mathrm{~nm}$. Figures $3(\mathrm{~d})$ and $3(\mathrm{f})$ show the expanded view of corresponding regions indicated in Figs. 3(c) and 3(e), respectively. We can see that Figs. 3(d) and 3(f) were focusing at different focal planes and were able to render high-resolution images of the sample at different planes.

At this point, it is worth noting that it is unclear if this Talbot illumination approach can provide resolution better than $1 \mu \mathrm{m}$. It may be possible to design the aperture geometry to diffract more light at large angles to help in tighter focus spot generation. The spot quality will also be degraded for thick scattering samples, especially when the focus spot is tighter.

In summary, we report a wide-FOV microscope based on Talbot pattern illumination. The wavelength of the laser source can be tuned to change the focal plane distance in order to obtain images at different focal planes. The elimination of mechanical scanning along the focal axis can be a significant advantage for fast scanning microscopy applications. The focal spot size was measured to be $\sim 1 \mu \mathrm{m}$ under a conventional microscope. Images of a USAF target and a mixed green algae microscope slide with an FOV of $6.4 \mathrm{~mm} \times 4.2 \mathrm{~mm}$ are acquired for demonstration of the system.

This work is supported by the United States Department of Defense under grant W81XWH-09-1-0051. The authors acknowledge Richard Cote and Ram Datar from the University of Miami for helpful discussions.

\section{References}

1. J. Ho, A. V. Parwani, D. M. Jukic, Y. Yagi, L. Anthony, and J. R. Gilbertson, Hum. Pathol. 37, 322 (2006).

2. M. Oheim, Br. J. Pharmacol. 152, 1 (2007).

3. W. Bishara, T. W. Su, A. F. Coskun, and A. Ozcan, Opt. Express 18, 11181 (2010).

4. M. G. Rojo, G. B. Garcia, C. P. Mateos, J. G. Garcia, and M. C. Vicente, Int. J. Surg. Pathol. 14, 285 (2006).

5. J. Wu, X. Cui, G. Zheng, Y. M. Wang, L. M. Lee, and C. Yang, Opt. Lett. 35, 2188 (2010).

6. R. Graf, J. Rietdorf, and T. Zimmermann, Adv. Biochem. Eng. Biotechnol. 95, 1311 (2005).

7. J. R. Leger and G. J. Swanson, Opt. Lett. 15, 288 (1990).

8. H. F. Talbot, Philos. Mag. 9, 401 (1836).

9. K. Patorski, Prog. Opt. 27, 1 (1989).

10. B. Besold and N. Lindlein, Opt. Eng. 36, 1099 (1997).

11. E. di Mambro, R. Haidar, N. Guerineau, and J. Primot, J. Opt. Soc. Am. A 21, 2276 (2004). 